\title{
Performance Indicators of Hospitals Affiliated To Ardabil University Of Medical Sciences In 2019
}

\author{
Hoseina Asadi ${ }^{1}$, Aghil Habibi Soola ${ }^{2 *}$, Raheleh Hamidi ${ }^{3}$, Ali Rezapour ${ }^{2}$, Solmaz Ershadifard ${ }^{2}$, Mahnaz Davari ${ }^{2}$ \\ 1. Research Committee, School Of Nursing and Midwifery, Ardabil University of Medical Sciences, Ardabil, Iran \\ 2. School Of Nursing and Midwifery, Ardabil University of Medical Sciences, Ardabil, Iran \\ 3. Ardabil University of Medical Sciences, Ardabil, Iran
}

Received: 28 February 2021

Accepted for publication: 3 May 2020

[EPub a head of print-17 May 2021]

Payesh: 2021; 20 (3): 285-293

\begin{abstract}
Objective (s): Hospital indicators are very important in indicating their performance. Indicators are used as a tool to compare the number of services, evaluate services, compare services with standards or compare them with previous years. The aim of this study was to determine performance indicators of hospitals in a number of teaching hospitals and comparing them with performance indicators of Ministry of Health and Medical Education, 2019

Methods: This was a cross-sectional study that descriptively examined the performance indicators of 12 hospitals affiliated to Ardabil University of Medical Sciences. The data were collected by referring to statistical unit of health deputy and analyzed using SPSS22 software.

Results: The ratio of active beds to fixed in 9 hospitals (75\%), bed performance ratio, admission ratio per bed and average length of stay in 10 hospitals $(83.3 \%)$ and bed turnover interval in 7 hospitals $(58.4 \%)$ were desirable. The bed occupancy ratio in 7 hospitals $(58.4 \%)$ was poor compared to the standards.

Conclusion: The performance indicators of the hospitals affiliated to Ardabil University of Medical Sciences were relatively good compared to the standards. However, there is a need for promotion and improvements. Therefore, it is necessary that managers take the necessary proceedings to increase hospitals' performance indicators.
\end{abstract}

Key Words: Performance indicators, hospitals, standard

\footnotetext{
* Corresponding author: School Of Nursing and Midwifery, Ardabil University of Medical Sciences

E-mail: habibiarums@gmail.com
} 
مقدمه: يكى از مهمترين عوامل نشان دهنده عملكرد بيمارستان، شاخص هاى بيمارستانى هستند. از شاخص هـا بـراى مقايسـه ميـزان خــدمات، ارزيـابى

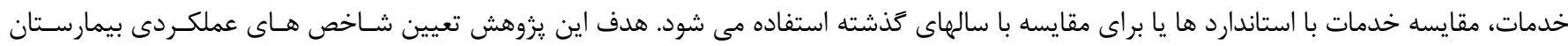

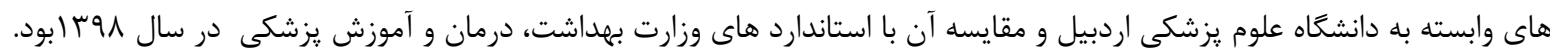

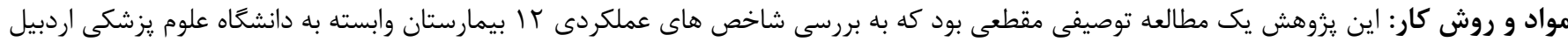

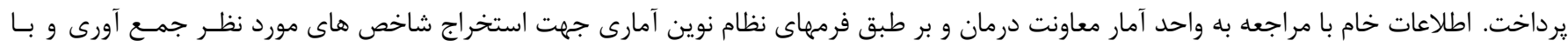

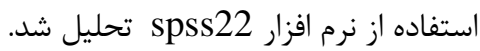

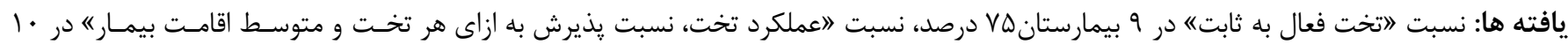

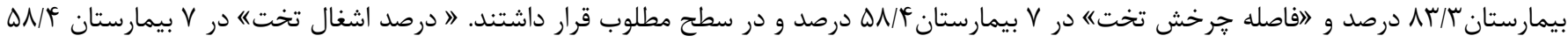

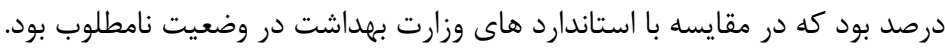
نتيجه كيرى: شاخص هاى عملكردى بيمارستان هاى وابسته به دانشكاه علوم يزشكى اردبيل نسبت به استاندارد در وضعيت نسبتاً مطلوبى قـرار دارنـد، امـاــا

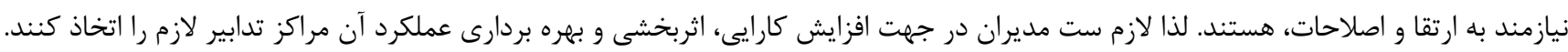
كليدوازه: شاخص هاى عملكردى، بيمارستان، استاندارد 
نخرش ها و يا موقعيت ها سر جشمه مى كيرند و قادر هستند ميـزان

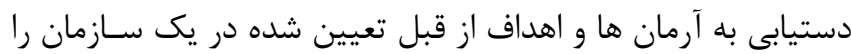

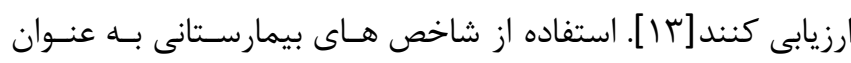

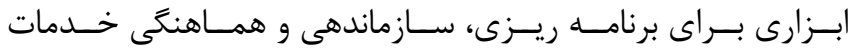

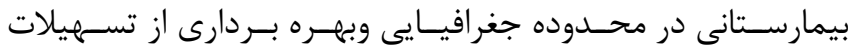

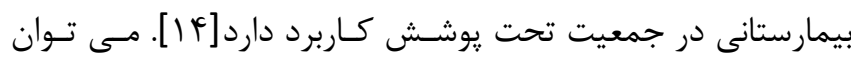

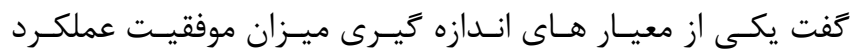

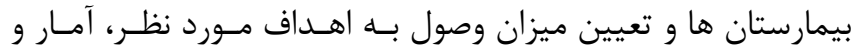

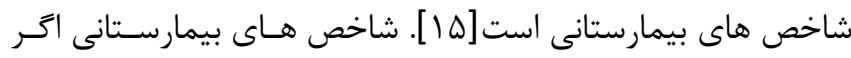

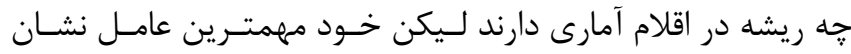
دهنده عملكرد بيمارستان است. از شاخص ها براى مقايسـه ميـزان

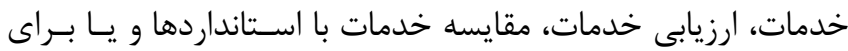

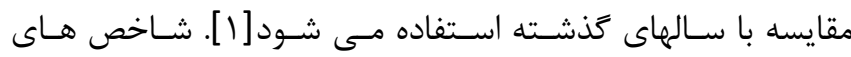

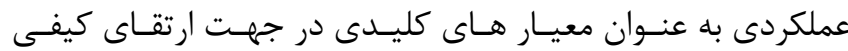

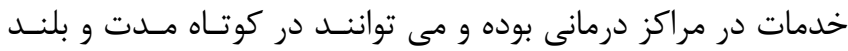

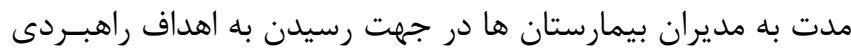

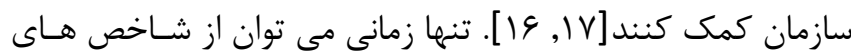

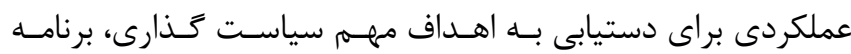

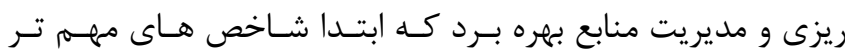

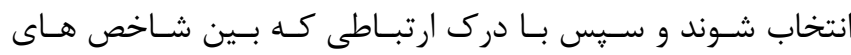

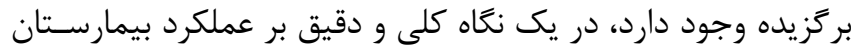

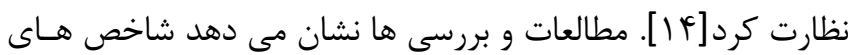

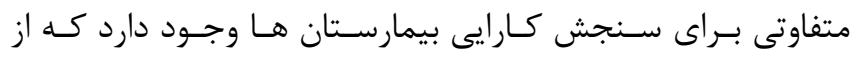

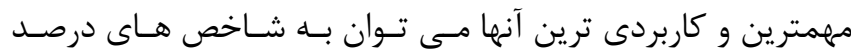

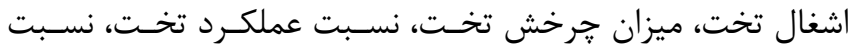
يذيرش به ازاى هر تخت، نسبت تخت فعال به ثابت و ميانگين مدت

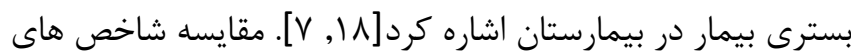

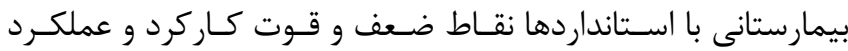

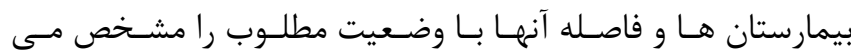

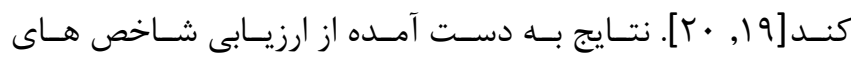

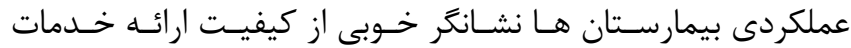
بهداشتى بوده و راهنماى خوبى براى برنامه ريزى و سياست كذارى آن

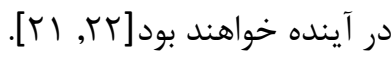
نتايج مطالعه طرسكى و همكاران در بررسى شاخص هاى عملكردى

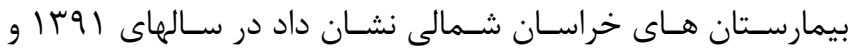

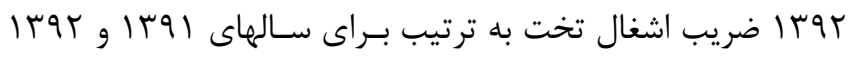

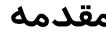

امـروزه ارتقــاى كيفيـت خــدمات درمـانى و دسـتيابى بـهـ بـالاترين

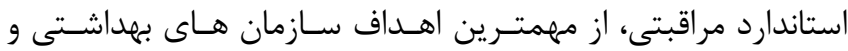

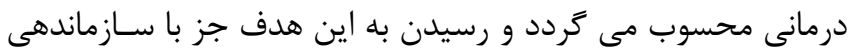

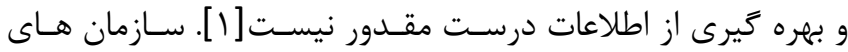

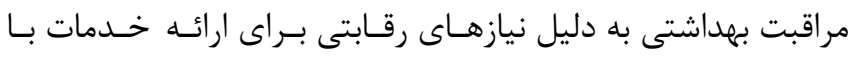

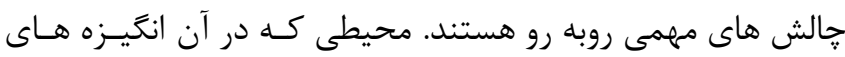

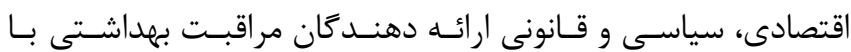
كنترل هزينه و حفظ كيفيت مواجه است، موجب مى شود كه تنها راه رسيدن به موفقيت مطلوب ارتقاى كيفيت با استفاده از اطلاعات

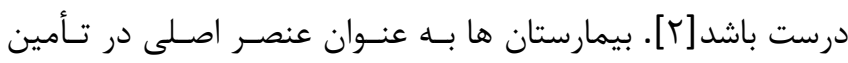

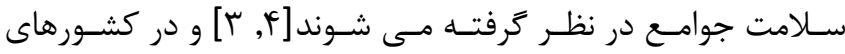

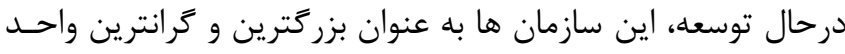

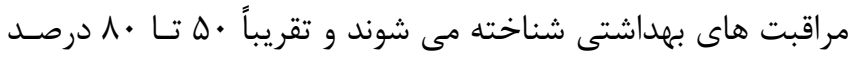

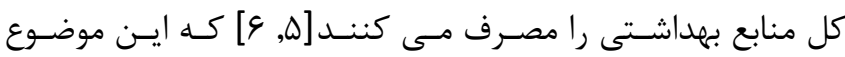

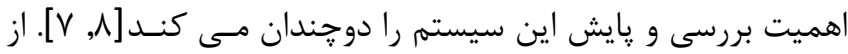

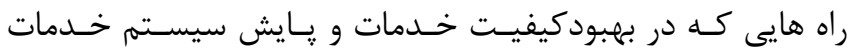
درمانى موثر است استانداردسازى و مقايسه شاخص هاى عملكـردى

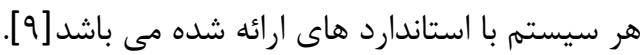

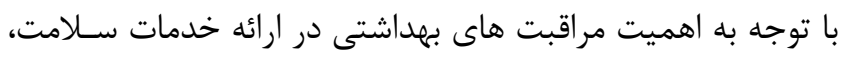

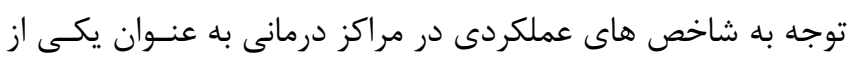

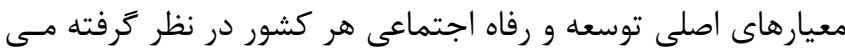

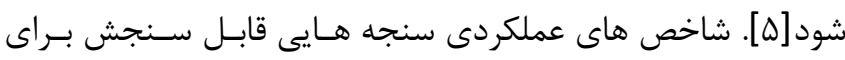

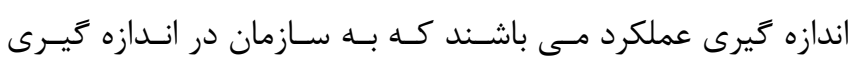
وتعيين ميزان ييشرفت در جهت تحقق اهداف كمك ميى كنيند. ايـن شاخص ها منعكس كننده عوامل مهـهم و حيـاتى موفقيـت سـازمان

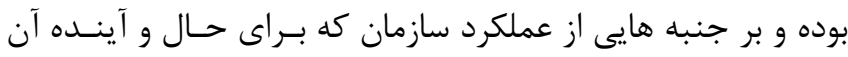

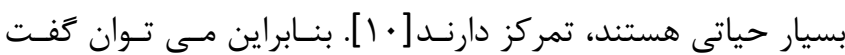

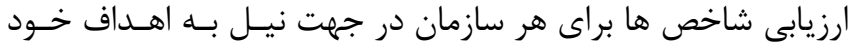

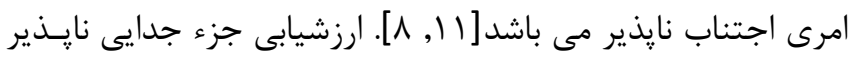

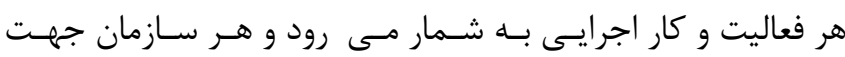

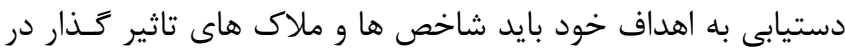

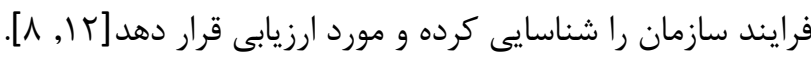

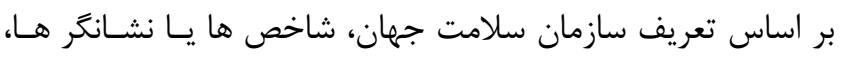
متغير هايى هستند كه به طور مستقيم يا غيــر مسـتقيم بــهـ انـدازه كيرى تغييرات كمك مى كنند[ [T]]. شاخص ها معمولا از نظريه ها، 
سببت يذيرش به ازاى هـر تخــت، نسـبت تخـت فعـال بــه ثابـت و

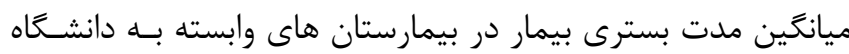

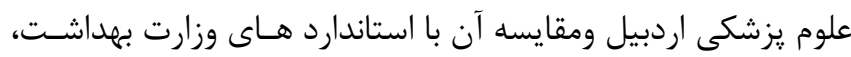

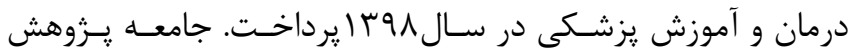
شـامل Y (بيمارستان(بيمارسـتان امـام خمينـى (ره)، امـام رضـا(ع)،

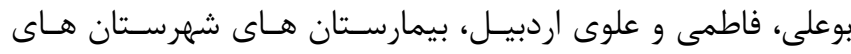

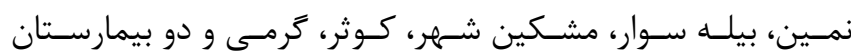
شهرستان يارس آباد اردبيل) وابسته به دانشگاه علوم يزشكى اردبيل

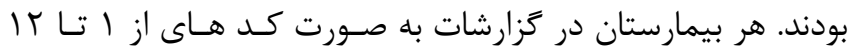

مشخص شده اند. ابزار يزوهش جهت گردآورى داده ها، جـى ليست اسـتاندارد وزارت

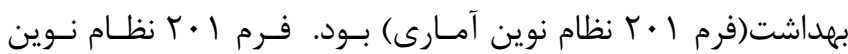

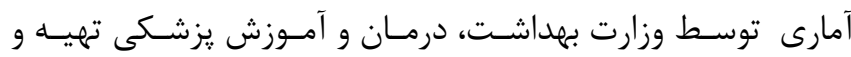
توسط كارشناسان، اعتبار آن تاييد شده است و در سطح كشـور، در كليه بيمارستان ها به صورت ماهيانه تكميل و وِ از تاييـد صـحت

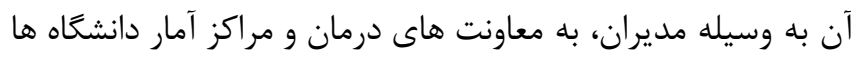

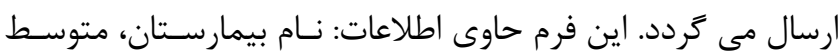
تخت فعال در ماه، تخت روز اشغالى، تعداد بيمار بسترى شده، تعداد

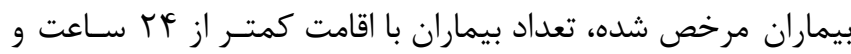
شاخص هاى عملكردى بيمارستان ها است. ضريب آلفـاى كرونبـاخ

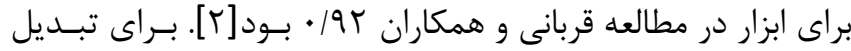

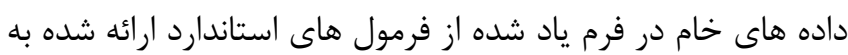
وسيله وزارت بهداشت و درمان از جمله موارد زير استفاده شد. هارد

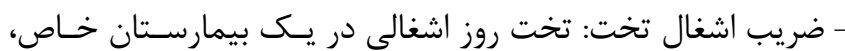

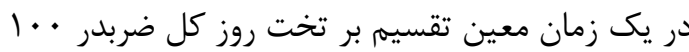

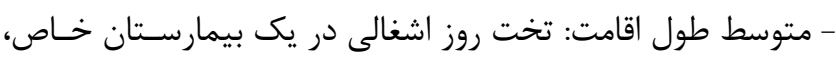
در يك زمان معين تقسيم بر مجموع بيماران فوت شـده و مـرخص شده در همان زمان - ميزان عملكرد تخت: تخت روز آماده در در يـك بيمارسـتان در يـك

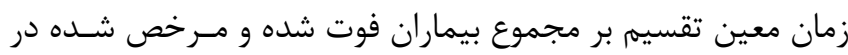
همان زمان

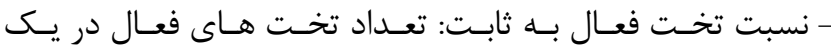
بيمارستان در زمان معين تقسيم بر تعداد تخت ثابت در همان زمان

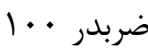
- نسبت يذيرش به ازاى هر تخت: تعداد بيماران يذيرش شده بـراى

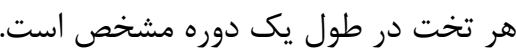

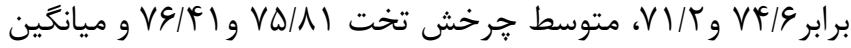

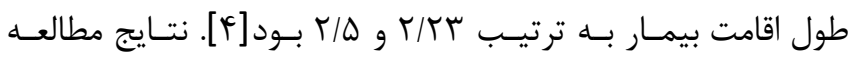

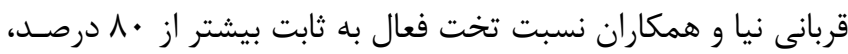
ضريب اشغال تخت بيشتر از • Vرصد، نسبت عملكرد تخت بيشتر

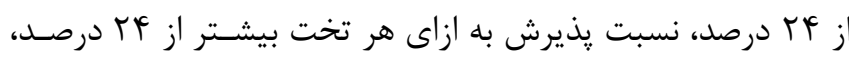

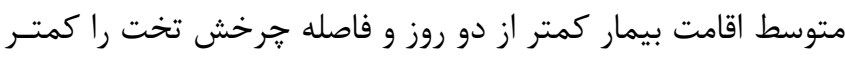

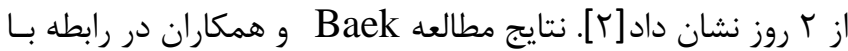

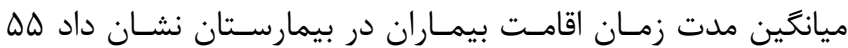
درصد از بيماران در مدت ع أ روز مرخص شده انـد. بيشـترين زمـان

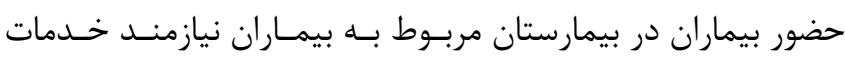
توانبخشى با مدت زمان نزديـك بـهـ 19 روز بـود. طيـف وسـيعى از

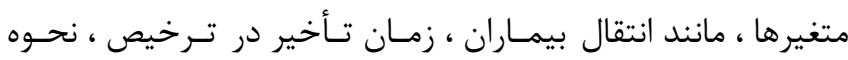

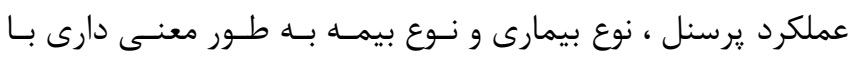

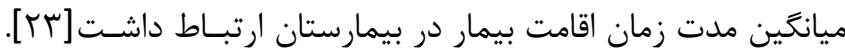

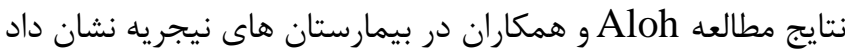

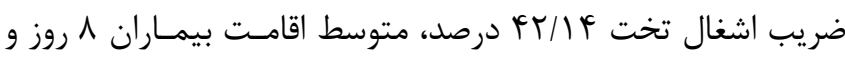

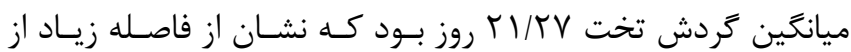

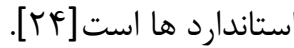

در اين :زروهش شاخص هاى درصد اشـغال تخـت، ميـزان جــرخش تخت، نسبت عملكرد تخت، نسبت يذيرش به ازاى هر تخت، نسـبت تخت فعال به ثابت و ميانگين مدت بسترى بيمار در بيمارسـتان بـا

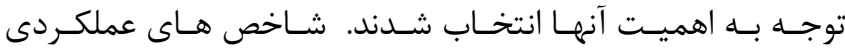

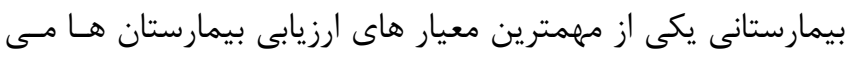

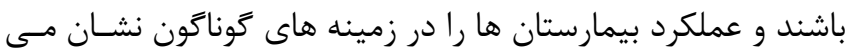
دهند، بنابراين توجه همه جانبه به اين شاخص ها از اهميت بـالايى برخوردار است. با توجه به عدم انجام مطالعه مشابه در سطح استان، اهميـت شـاخص هــاى عملكــردى بيمارسـتانى در تعيـين ميـزان دستيابى به اهداف سازمانى و همجنين تعيين وضعيت شاخص هاى

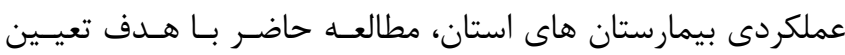
شاخص هاى عملكردى بيمارستان هاى وابسـته بـهـ دانشـعاه علـوم

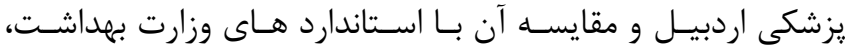

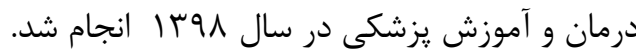

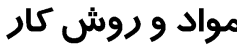

اين يزوهش يك مطالعه توصيفى مقطعى بود كه به بررسى شـاخص هاى درصد اشغال تخت، ميزان جرخش تخت، نسبت عملى عملكرد تخت، 
بيشترين ميـزان ضـريب اشـغال تخــت (9/9/9

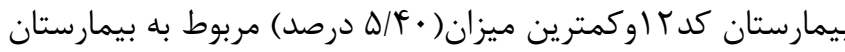

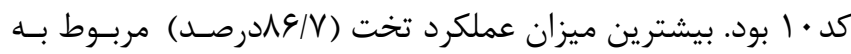

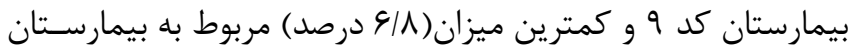
كد • إبود. بيشترين ميزان يذيرش به ازاى هـر تخــت ( • (ادرصـد)

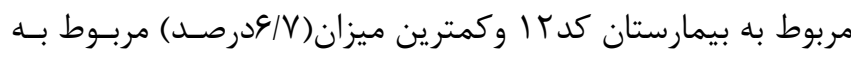

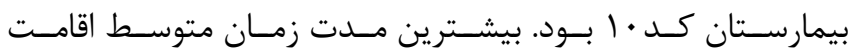

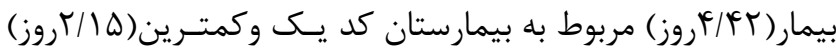

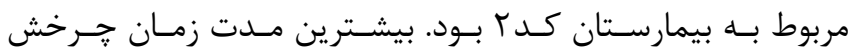

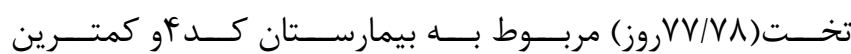

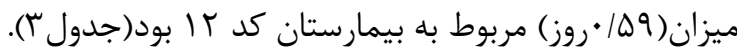
نتايج نشان داد نسبت تخت فعال به ثابت در مقايسه با استاندارد هـا درج بيمارستان (VVDدرصد)در سطح مطلوب قرار دارد. درصد اشـغال

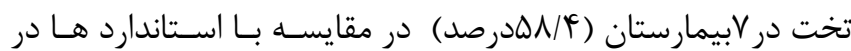
وضعيت نامطلوب بود. نسبت عملكرد تخت و نسبت يذيرش به ازاى

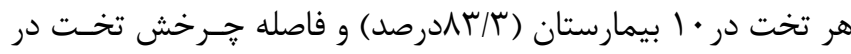

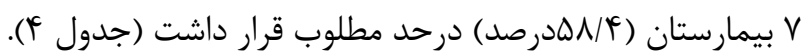

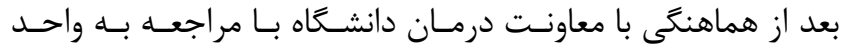
فناورى اطلاعات، داده ها استخراج گرديد. شاخص هـاى عملكـردى بـى

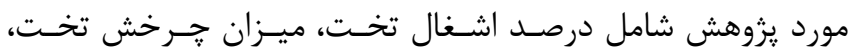

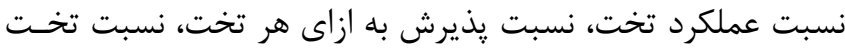
فعال به ثابت و ميانخين مدت بسترى بيماردر بيمارسـتان بـود. داده

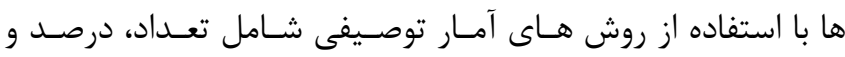
ميانگين به كمك نرم افزار آمارى SPSS 22 تحليل شـد. شـاخص

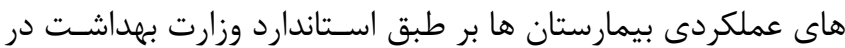

سه سطح مطلوب، متوسط و نامطلوب تقسيم شده اند(جدول ( ).

يافتهها

بيمارستان كد يكى با تعـداد هضه تخــت مصـوب بـا متوسـط تعـداد

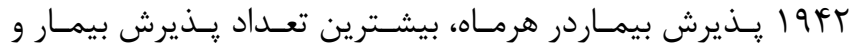

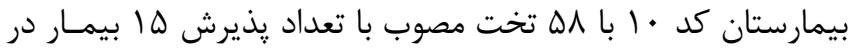

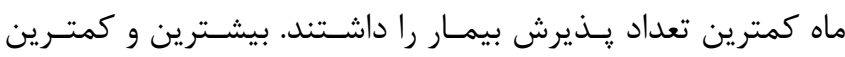

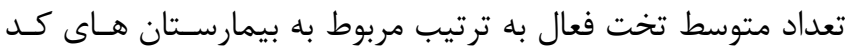
يك و جهار بود(جدول r).

جدول ا: استاندارد شاخص هاى عملكردى بيمارستان ها طبق اعلام وزارت بهداشت، درمان و آموزش يزشكى

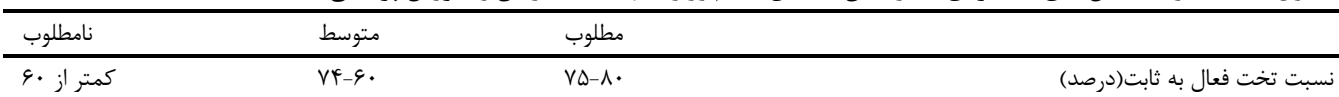

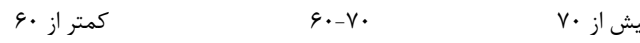

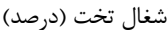

بيش از بيث از كمتر از

IV بيش از TFF
نسبت عملكرد تخت (درصد)

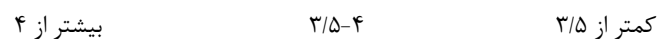

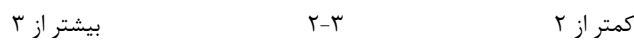
فاصله خرخش تخت(روز)

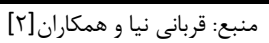

جدول r: مشخصات بيمارستان ها

\begin{tabular}{|c|c|c|c|}
\hline متوسط تخت فعال & تعداد تخت مصوب & متوسط يذيرش بيمار درماه & كد بيمارستان \\
\hline$\varphi \cdot \varphi / \Delta \Lambda$ & $\Delta \Delta \Delta$ & 1945 & 1 \\
\hline ११/v & $1 .$. & VVA & r \\
\hline १1 & 11. & DIf & $r$ \\
\hline rq & qr & rI & $\varphi$ \\
\hline 149 & 10. & val & $\Delta$ \\
\hline$\Delta V$ & $\Delta r$ & 199 & 9 \\
\hline vi & 114 & rfF & V \\
\hline rIT & Tr. & IrGY & $\wedge$ \\
\hline rrI & Tr. & ifrr & 9 \\
\hline rr & $\Delta \Lambda$ & 10 & 1. \\
\hline 1.0 & १V & Frt & 11 \\
\hline $1 \Delta T$ & $1 \Delta \Delta$ & $1 \% 91$ & Ir \\
\hline
\end{tabular}


If.. سال بيستم، شماره سوم، خرداد ـ تير

نشريه يُوهشكده علوم بهداشتى جهادانشعاهى

جدول ז: وضعيت شاخص هاى عملكردى بيمارستان هاى دانشعًاه علوم يزشكى اردبيل در سال ه1

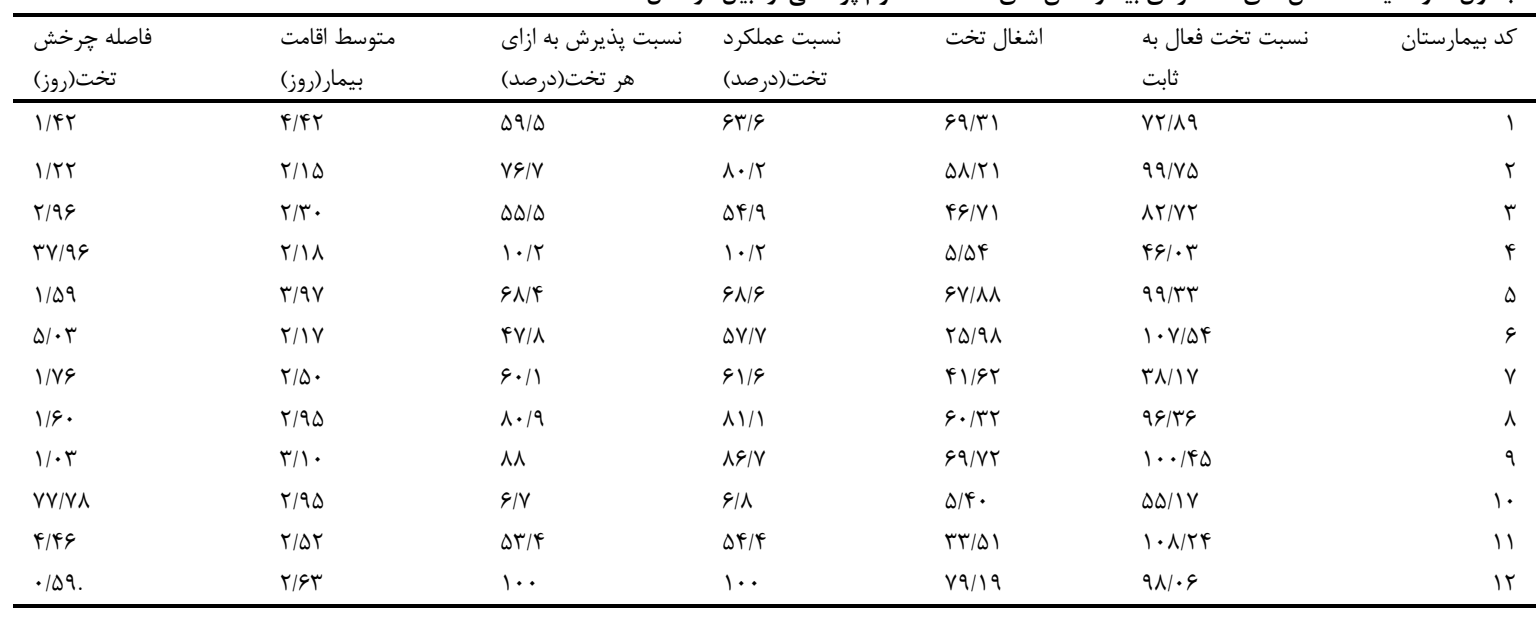

جدول f: وضعيت شاخص هاى عملكردى بيمارستان هاى دانشعاه علوم يزشكى اردبيل در سال ^هץ ا در مقايسه با استاندارد ها

\begin{tabular}{|c|c|c|c|c|c|c|}
\hline \multicolumn{2}{|c|}{ 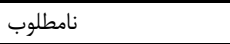 } & \multicolumn{2}{|c|}{ متوسط } & \multicolumn{2}{|l|}{ مطلوب } & \\
\hline درصد & تعداد & 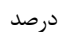 & تعداد & 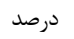 & تعداد & \\
\hline $19 / V$ & $r$ & $\Lambda / r$ & 1 & $v_{\Delta}$ & 9 & نسبت تخت فعال به ثابت(درصد) \\
\hline$\Delta \Lambda / \mathcal{\epsilon}$ & v & 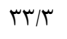 & r & $\Lambda / r$ & 1 & اشغال تخت (درصد) \\
\hline $19 / V$ & r & . & . & N & 1. & نسبت عملكرد تخت(درصد) \\
\hline $19 / \mathrm{V}$ & r & . & . & $\Lambda \mu / r$ & 1. & نسبت پذيرش به ازاى هر تخت(درصد) \\
\hline$\Lambda / r$ & 1 & $\Lambda / r$ & 1 & $\Lambda \mu / r$ & $1 \cdot$ & متوسط اقامت بيمار(روز) \\
\hline ז/r & r & $\Lambda / r$ & 1 & $\Delta \Lambda / F$ & $v$ & فاصله خرخش تخت(روز) \\
\hline
\end{tabular}

بيمارستان ها در وضعيت نامطلوب قرار داشت. در مطالعه ارزمانى و و دران

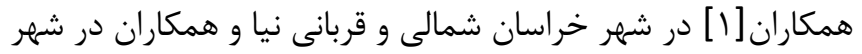

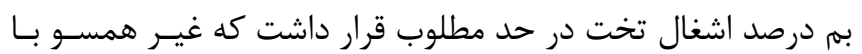

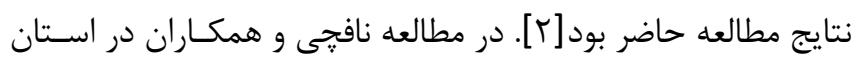

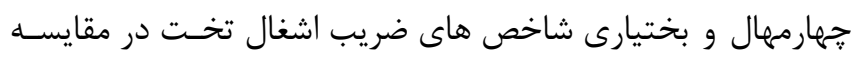

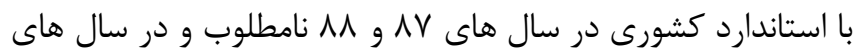

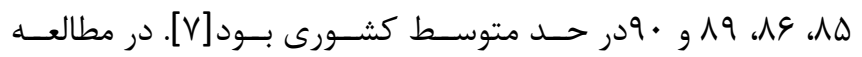

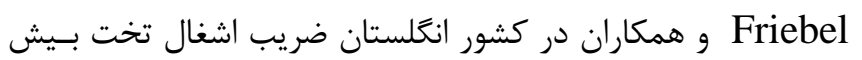

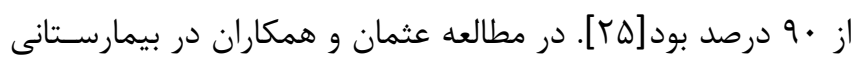

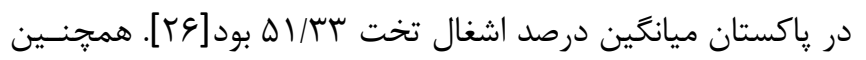

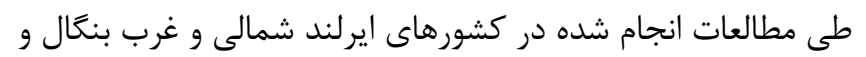

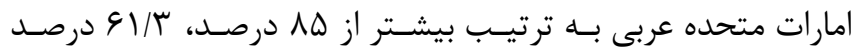

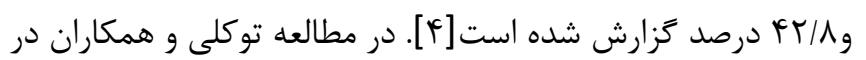

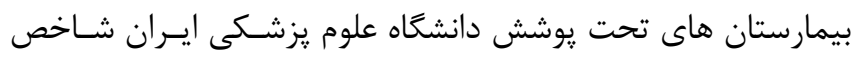

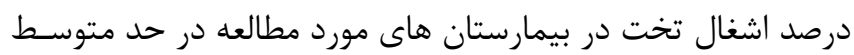

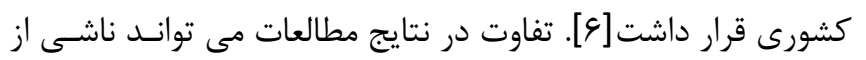

بتث و نتيجه كيرى

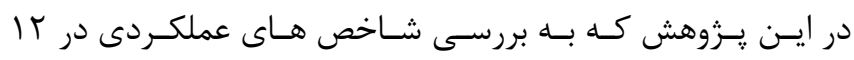

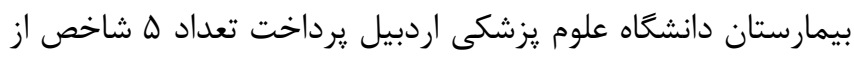

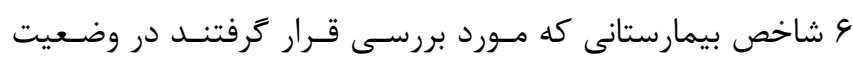

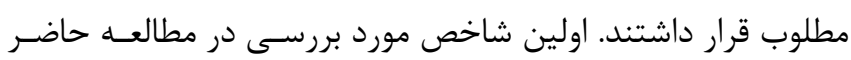

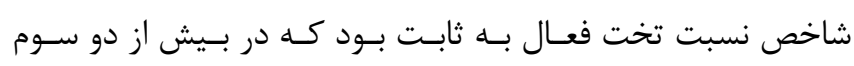

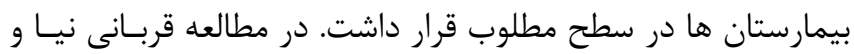

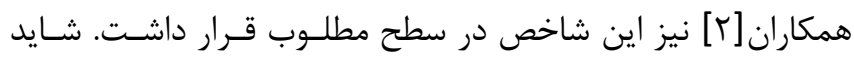

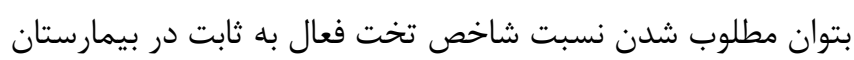

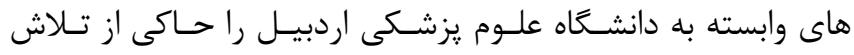

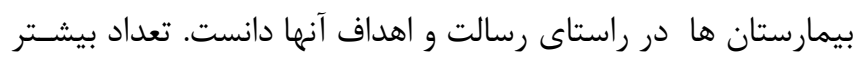

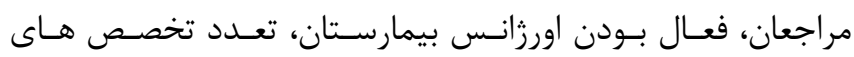

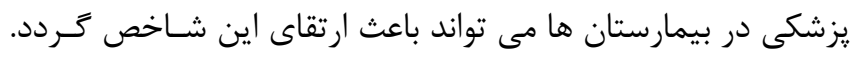

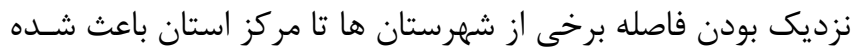

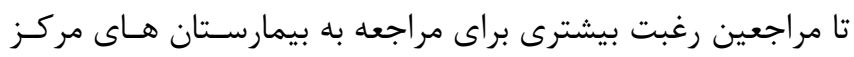

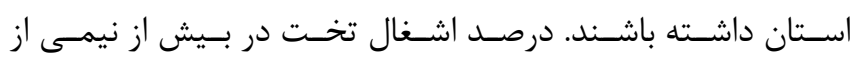


وضعيت مطلوب اين شاخص را به دليل فعاليت صحيح بيمارسـتان

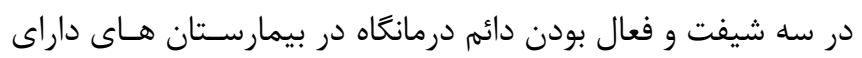

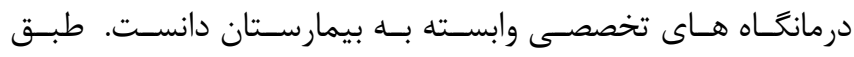

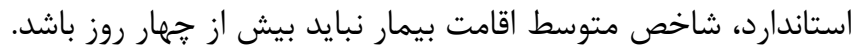

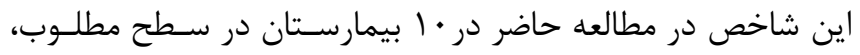

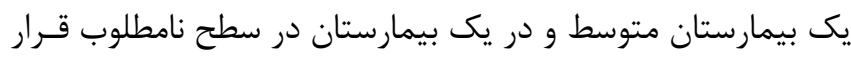

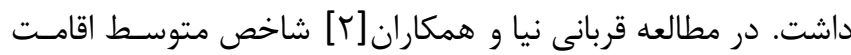

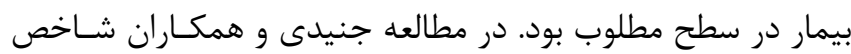

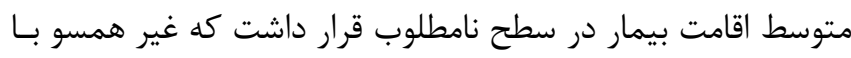

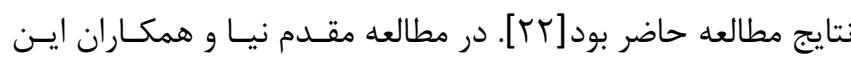

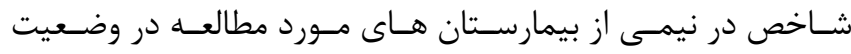

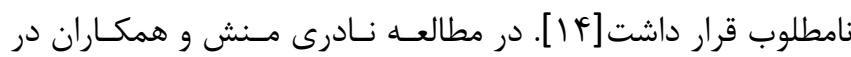
شهر تهران شاخص متوسط اقامت بيمار در بيمارستان هاى آموزشى دانى

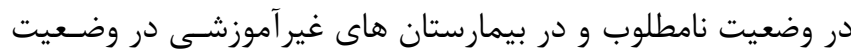

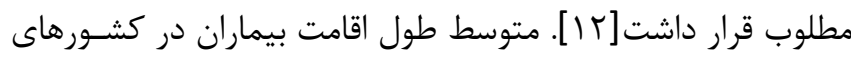
OCED

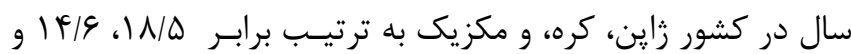

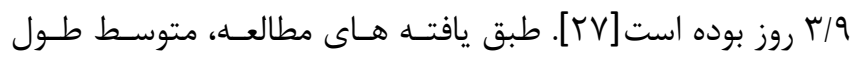

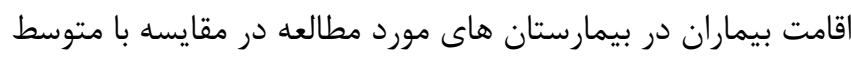

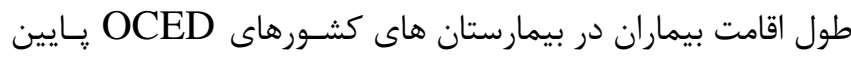

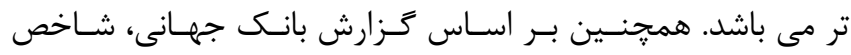

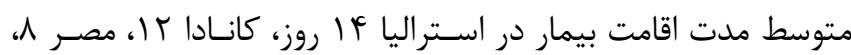

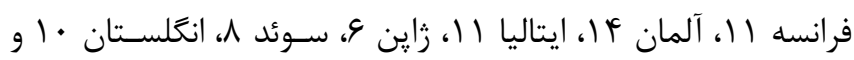

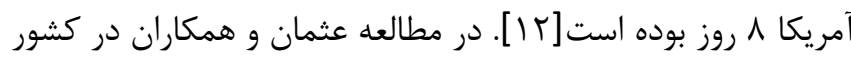

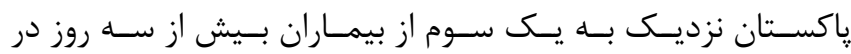

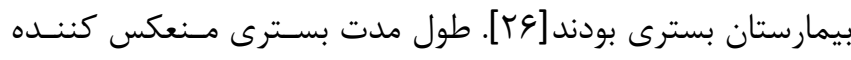

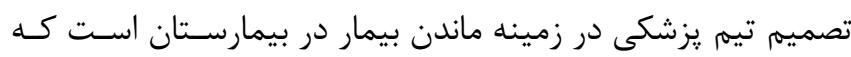

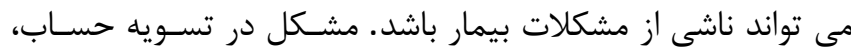

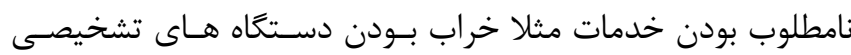

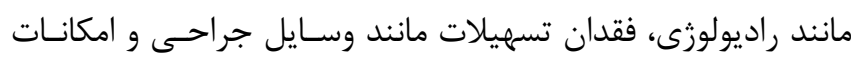

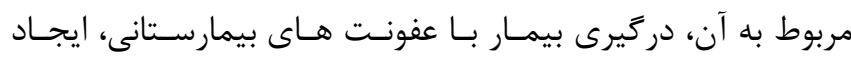

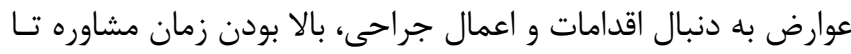

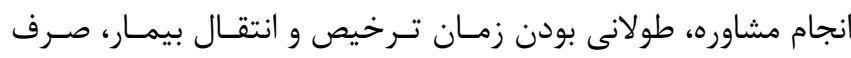

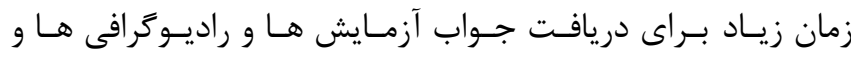

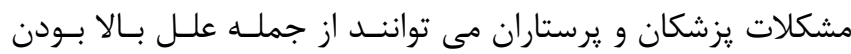

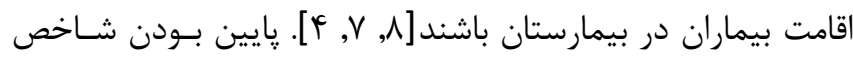

نوع مراكز درمانى، حضور منظم يزشك در اورزانس، يوشش بيمه ايى

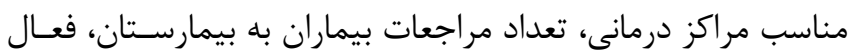

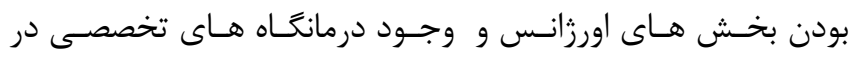

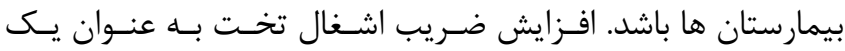

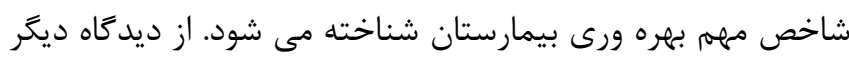

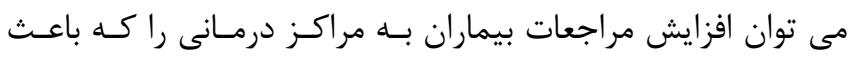

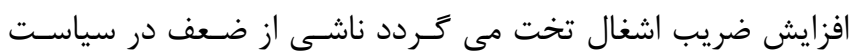

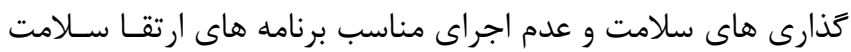
جامعه محور نيز دانست.

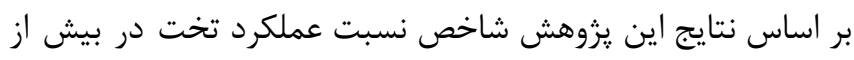

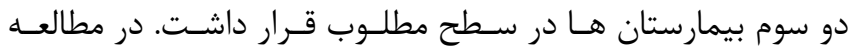

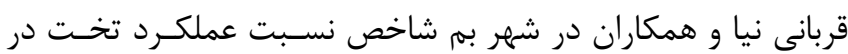

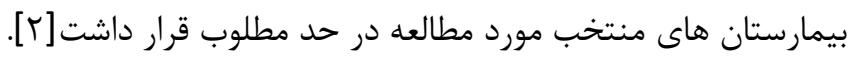

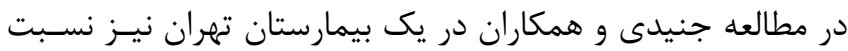

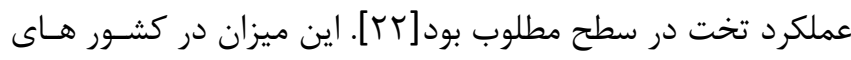

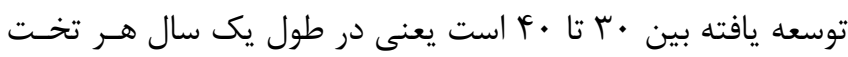

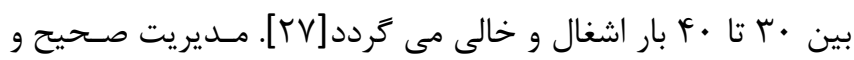
بهبود فرآيند يذيرش و ترخيص بيمارستان مى تواند از عوامل تـاثير

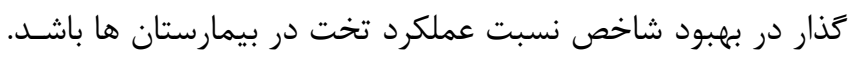

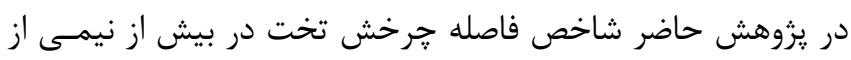

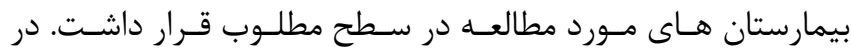

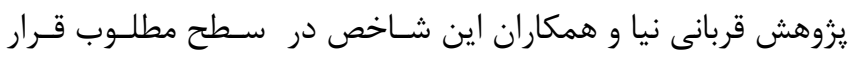

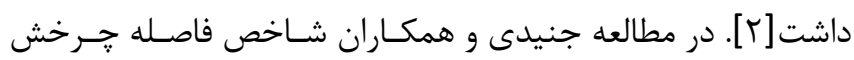

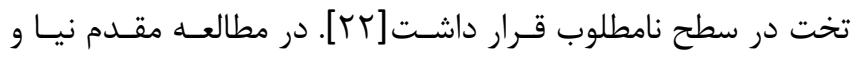

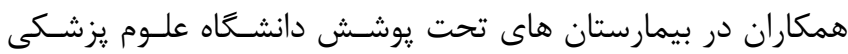

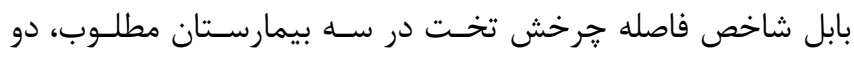

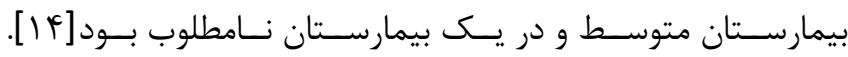

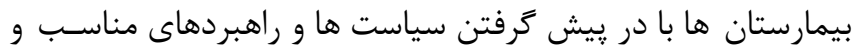

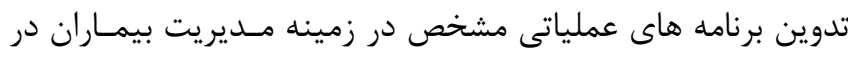

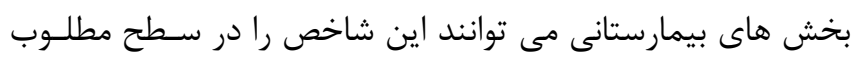

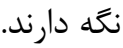

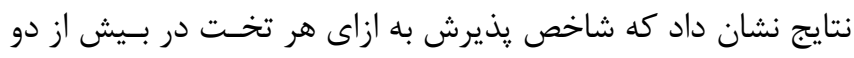

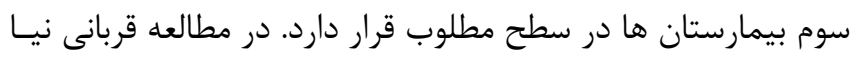

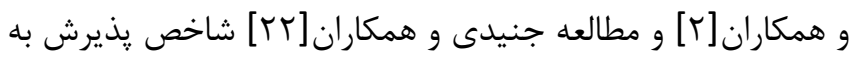

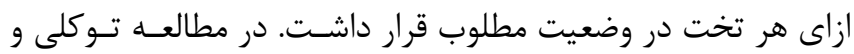
همكاران نيز اين شاخص در سطح مطلوب قرار داشت. شـايد بتـوان 
نيز عوامل موثر بر تغييرات هر يك از شـاخص هــاى بيمارسـتانى در

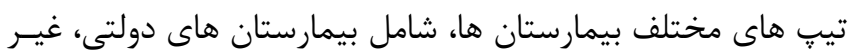

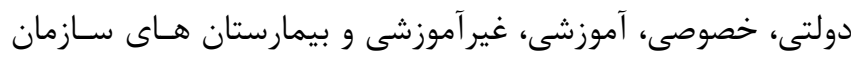

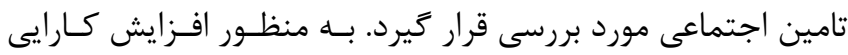
بيمارستان ها و جلوگيرى از اتلاف منابع، الخو بردارى از بيمارسـتان هاى كارآمد مد نظر مديران و سياست كذران بهداشت و و درمان قرار كيرد.

\section{سهم نويسند}

حسين اسدى: مجرى اصلى طرح، ارائه طرح، تهيه مقاله سولماز ارشادى فرد: مجرى اصلى طرح، ارائه طرح، تهيه مقاله عقيل حبيبى: استاد راهنما و نويسنده مسئول مبرى راحله حميدى: همكارى در تجزيه و تحليل آمارى، كمك در نكارش

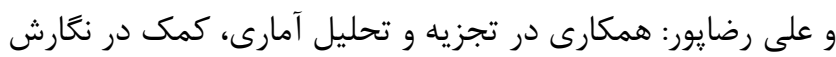
مهناز داورى: كمك در طراحى يزوهش و نغارش مقاله

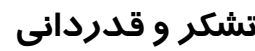

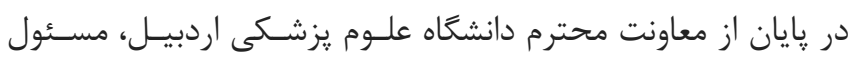
فناورى آمار و اطلاعات دانشگاه علوم يزشكى اردبيل كه در تهيه اين

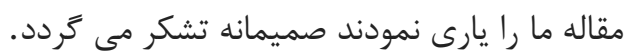

\section{منابع}

1. Arzemani M, Pournaghi SJ, Syed katooli SM, Jafakesh moghadam A. The Comparison of performance indicators in educational hospitals of North Khorasan Universities of Medical Sciences with. Journal of North Khorasan University of Medical Sciences 2013;4:513-521

2. Ghorbani R, Ahmadian K, Ranjbar Z. To Compare the Performance Indexes of Selected Hospital with National Standards. Journal of Health Promotion Managment 2017;7:1-7

3. Khammarnia M,AliNejad Ranjbar A ,Bakhshi M. Comparison of Performance Indicators of Zahedan Public Hospitals Before and After Implementation of Hospital Accreditation Plan. Scientific Journal of School of Public Health and Institute of Public Health Research 2019;16:145-56

4. Toroski M, Golmakani E, Sodagar HR, Hosseini SH, Rajabzadeh R, Borhaninejad VR, et al. Survey of bed efficiency for hospitals of North Khorasan University of Medical Sciences by using standard functional criteria of the ministry of health. Journal of
متوسط اقامت بيمار در بيمارسـتان هــاى مـورد مطالعـه مـى توانـد

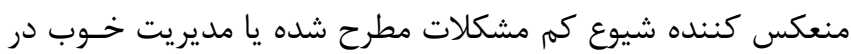
اين بيمارستان ها باشد.

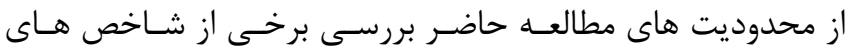

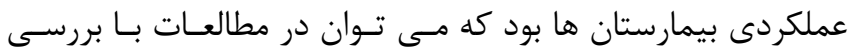

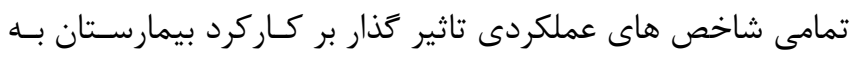
نتايج بهترى دست يافت. همجنين مى توانى تهان بابر رسى و مقايسه نتايج

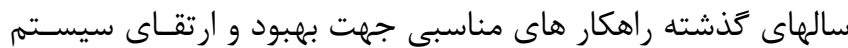
درمانى ارائه كرد. نتايج مطالعه نشان داد كه وضعيت اكثـر شـاخص هــاى عملكـردى بيمارستان هاى وابسته به دانشگاه علوم يزشكى اردبيل بجز شاخص

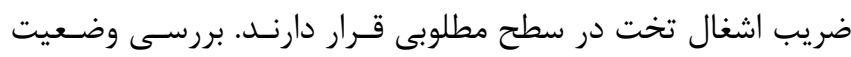

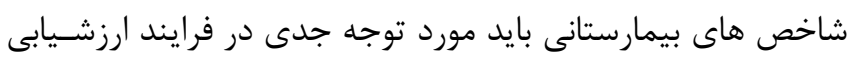

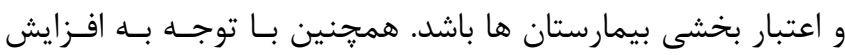

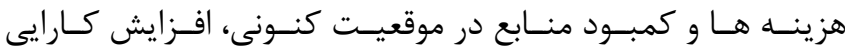

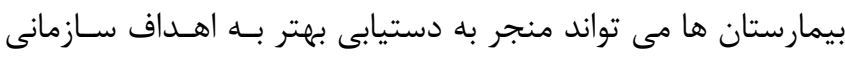

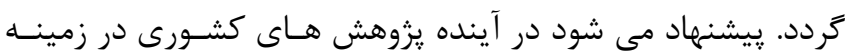

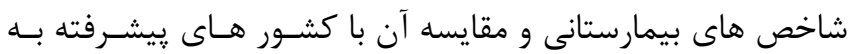
منظور دستيابى به استاندارد هاى ملى در اين زمينــه انجـام شـود و

North Khorasan University of Medical Sciences 2014;6:637-644

5. Lotfi F, Kalhor R, Bastani P, Shaarbafchi Zadeh N, Eslamian M, Dehghani MR, et al. Various indicators for the assessment of hospitals' performance status: differences and similarities. Iranian Red Crescent Medical Journal 2014;16: 12950

6. Tavakoli N, Rezapour A, Gholami Somarin A, Ameri H, Mohammadi R, Bagheri Faradonbeh R. Performance Indicators of Hospitals Affiliated to Iran University of Medical Sciences and Comparing them with Performance Indicators of Ministry of Health and Medical Education. Management Strategies in Health System 2020; 4: 328-36

7. Reisi-Nafchy M, Drees F, Mirzaeian R. Assessment of performance indicators in Hospitals University of Medical Sciences based on the standards of the Ministry of Health. Journal of Shahrekord University of Medical Sciences 2014; 15: 60-67

8. Samadi S, Nasiri Pour AA, Kazem Nejad A, Raeesi Dehkordi P. Evaluation of Indicators and Standards of 
Accreditation Programs in Military Health Centers: A Narrative Review Study. Journal Of Pilice Medicine 2020;9:43-52

9. Tofighi Sh, Fallah MS, Khajeh Azad M. Quality evaluation of knowledge management in a military hospital based on the Baldrige excellence model. Iranian Journal of Military Medicine 2012;13:213-6

10. Mousavi Rigi A, Bahrami M, Montazerolfaraj R, Dehghani Tafti A, Dorahaki M, Barati O. Reviews and Comparisons of Hospital Performance Indicators Before and After the Implementation of the Healthcare Reform Package Design Therapeutic Hospitals of Bushehr University of Medical Sciences. Journal of Tolooebehdasht 2016;15:107-19

11. Vafaee-najar A, Ebrahimipour H, Houshmand E, Zomorrodi-niat $\mathrm{H}$. Identification and Prioritization of Hospital Performance Evaluation Indices by Combination of Balanced Scorecard and Delphi (Montaserieh Hospital). Navidno journal 2018;21:1-1

12. Naderi Manesh KH, Nasiripour AM, Masoudi Asl SH, Babouei AM, Ostovar R, Rezanjad MT. The Study of the Status of Performance Indicators in Selected Educational and Non-Educational Hospitals of Tehran Province. Armaghane-danesh, Yasuj University of Medical Sciences Journal 2020;25:104117

13. Meraji M, Sajjadi mirzaee S,Taghizade Manouchehri Monazah F. Comparing emergency department performance indicators in Trauma centers. Journal of Health Administration 2019;22:67-77

14. Moghadamnia AA, Jahan MA, Bijani A, Yaminfirooz M, Naghshineh A, Mohammadnia K. Evaluation of Performance Indicators and Frequency of Patient Referrals in the Hospitals Affiliated To Babol University of Medical Sciences in 2013. Journal of Babol University of Medical Sciences 2016;18:61-9

15. Mohammadi M, Safdari R, Gholamhosseini L, Shamsabadi A. Survey of Documentation, accuracy and precision of hospital indices in selected educational hospital of Tehran University of Medical Sciences, 2014. Paramedical Sciences and Military Health 2014;9:21-7

16. Ebadi fard azar F, Choopani A, Saberi anari H, Mohammadi N, Arkian SH, Choopani F. Comparing performance indicators of obstetric and gynecology ward with expected limits of indicators. Journal of Hospital 2011;11:51-61
17. Rahimi H, Khammar-nia M, Kavosi Z, Eslahi M. Indicators of Hospital Performance Evaluation: A Systematic Review. International Journal of Hospital Research 2014;3:199-208

18. Sharif M, Motaghi M, Sehhat M. Compression of clinical indicators before and after the implementation of clinical governance and accreditation programs in Beheshti Hospital - 2012 .Beyhagh 2013;20:14-25

19. Baratloo A, Rahmati F, Forouzanfar MM, Hashemi B, Motamedi M, Safari S. Evaluation of Performance Indexes of Emergency Department. Iranian Journal of Emergency medicine 2014;2:33-8

20. Ghazizadeh J, Partovi Y, Alidoost S, Kavakebi N. Performance indicators of hospitals affiliated to Health Network in East Azerbaijan before and after health reform. Payesh 2018;17:217-26

21. Gholamzadeh Nikjoo R, Jabbari Beyrami $H$, Jannati A, Asghari Jaafarabadi M. Selecting Hospital's Key Performance Indicators, Using Analytic Hierarchy Process Technique. Journal of Community Health Research 2013;2:30-8

22. Jonaidi N, Sadeghi M, Izadi $M$, Ranjbar R. Comparison of performance indicators in one of hospitals of Tehran with national standards. Iranian Journal of Military Medicine 2011;12:223-8

23. Baek H, Cho M, Kim S, Hwang H, Song M, Yoo $\mathrm{S}$. Analysis of length of hospital stay using electronic health records: A statistical and data mining approach. PLoS ONE 2018;13:e019590.

24. Aloh H E, Onwujekwe OE, Aloh OG, Nweke CJ. Is bed turnover rate a good metric for hospital scale efficiency? A measure of resource utilization rate for hospitals in Southeast Nigeria. Cost Effectiveness and Resource Allocation 2020;18:1-8

25. Friebela R, Rebecca F, Deenyc SR, Gardnerc T, Molloyd AE, Steventon A. The implications of high bed occupancy rates on readmission rates in England: A longitudinal study. Health Policy 2019;123:765-72

26. Gulzar U, Khalida NM, Shazia Sh. Bed occupancy rate and length of stay of patients in medical and allied wards of a tertiary care hospital. Journal of Ayub Medical College Abbottabad 2015;27:367-70

28. OECD (2013), Education at a Glance 2013: OECD Indicators, OECD Publishing. Available at: http://dx.doi.org/10.1787/eag-2013-en 